\title{
Cryogenic Angle Measurement at NASA Langley
}

\author{
Kenneth G. Toro*, and Peter A. Parker ${ }^{\dagger}$ \\ NASA Langley Research Center, Hampton, Virginia, 23681, USA
}

\begin{abstract}
NASA's National Transonic Facility is one of the few cryogenic wind-tunnel facilities in the world, where tunnel conditions may reach $-250^{\circ} \mathrm{F}$. Model angle of attack (AoA) at NTF is typically measured by an on-board accelerometer package that is exposed to the cryogenic tunnel conditions. These NTF AoA packages house two Q-Flex accelerometers in a compact heated environment in order to maintain constant temperature of the sensors for stability. Recently, drift in sensor output was observed in one of the NTF AoA packages. The drift is correlated with the AoA package temperature and cryogenic soak time. This paper studies the temperature and time dependent behavior of the NTF AoA packages, and reviews their design and calibration. Recommended improvements to the AoA packages are made to improve the stability of the model angle of attack measurements at cryogenic conditions.
\end{abstract}

\section{Introduction}

NASA's National Transonic Facility (NTF) is one of the premier cryogenic wind-tunnel facilities in the world. ${ }^{1}$ NTF was specifically designed to achieve flight Reynolds numbers of transportation aircraft around transonic flight conditions. In order to achieve ground test with high Reynolds numbers, the circuit is pressurized and operates at temperature ranges of $-320^{\circ} \mathrm{F}$ to $160^{\circ} \mathrm{F}$. At these conditions, wind-tunnel model instrumentation are subjected to NTF temperature ranges, while they are expected to maintain room temperature performance. Most precision instrumentation are in some manner affected by temperature, where proper analysis is required to ensure proper operation. It has been shown that force balances and Q-Flex accelerometers are sensitive to temperature changes. ${ }^{2,3}$

Accelerometers have been used for angle of attack (AoA) measurement since the 1940's and has steadily improved in performance through technology improvements. ${ }^{4}$ Currently at NTF, Q-Flex accelerometers are the primary method of on-board AoA measurement, where a sensing element on pendulous flexures are made from fused quartz. Q-Flex or similar accelerometers have been implemented at other facilities around the globe for AoA measurements. ${ }^{5,6}$

NTF typically conducts performance testing of transport aircraft due to the high Reynolds capability that demands high AoA accuracies. It is widely accepted that for performance wind-tunnel test require AoA measurement accuracy be on the order of $0.01^{\circ}$ to measure a 0.0001 change in the drag coefficient. ${ }^{7,8}$ Ample testing of Q-Flex accelerometers have demonstrated high accuracy and repeatability within the desired $0.01^{\circ}$ requirement. It was shown by Crawford that to maximize the performance of Q-Flex sensors, either temperature corrections should be applied or that the sensor is maintained at a temperature. ${ }^{3}$

NTF AoA packages are designed to cope with this environment by placing the instruments in a compact thermal chamber, where a temperature of $160^{\circ} \mathrm{F}$ is maintained. ${ }^{4}$ This thermal chamber is composed of a closed, high-density foam, cylinder containing a heated aluminum enclosure where one or two Q-Flex sensors are placed. This heated environment would avoid the need for temperature corrections, thus yielding stable angle measurements. However, recent testing revealed detectable shifts in sensor output on several AoA packages. This paper focuses on reviewing the design and manufacturing of the NTF AoA packages, and an analysis into temperature dependent shifts in the sensors behavior.

\footnotetext{
${ }^{*}$ Research Engineer, System Engineering and Engineering Methods, NASA Langley Research Center

$\dagger$ Team Lead, Advanced Measurement and Data Systems, NASA Langley Research Center
} 


\section{Background}

\section{A. AoA Package Design}

The typical onboard AoA packaged used for full-span model testing at NTF is built with two internal Q-Flex sensors. An illustration of the typical package design is shown in Figure 1, where two Q-Flex sensors are adjoined back-to-back to provide redundant measurement. Elastic pads are placed at specific locations on the joined Q-Flexs to mitigate vibration effects on the sensor and hosed in an inner aluminum case. To stabilize the temperature of the Q-Flex sensors, heaters wrap the inner housing to maintain a interior temperature of $160^{\circ} \mathrm{F}$. To reduce the influence of the tunnel temperature on the sensors, the heated inner hosing is encased in insulation foam.

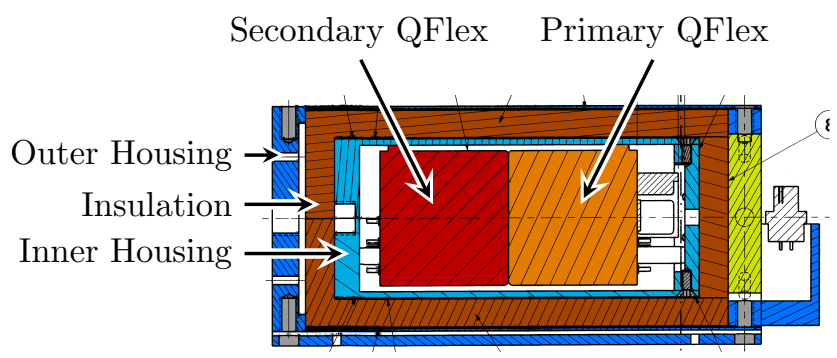

Figure 1: NTF Two Q-Flex Package Design

\section{B. Motivation}

A previous test entry using the NTF203-1 AoA package exhibited significant drift of the estimated angles between the two internal Q-Flex sensors as shown in Figure 2. The two temperature measurements were taken on two different days at cryogenic temperatures. Trend found in both runs have similar slopes, which indicates that the trends are not due to random drift and are repeatable between units. It is important to note that the largest difference between the pimary and secondary sensors does not deviate more than $0.01^{\circ}$.

Explain what Pitch and PitchM are

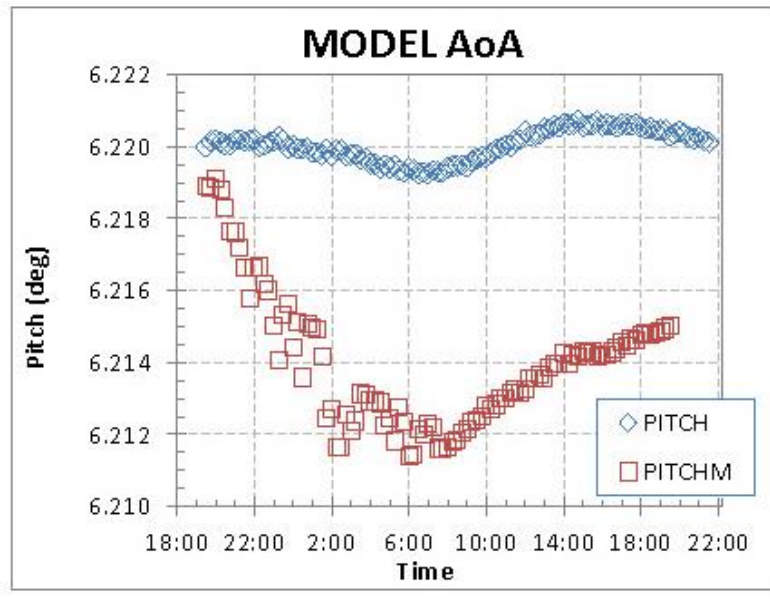

(a) Temperature Drift Run 1

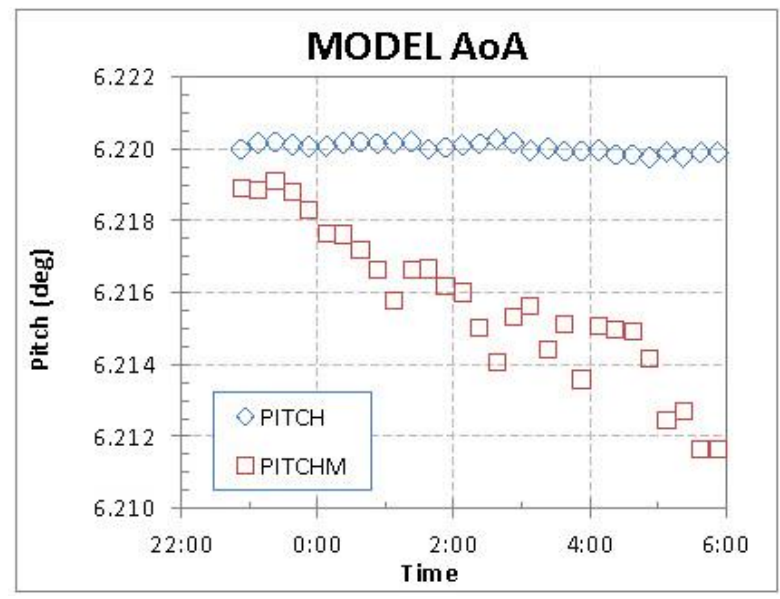

(b) Temperature Drift Run 2

Figure 2: Cryogenic Temperature Drift Runs of NTF203-1

Similar NTF AoA packages, such as the NTF204-1, have not exhibited sensor drifts of the magnitudes seen from the NTF203-1. This indicates that the NTF203-1 is not in family with other NTF AoA packages in terms of performance. The drifts found in the NTF203-1 warrant cryogenic test to identify the causality of the observed sensor performance, and corrective measures to bring the package back into family. 


\section{NTF203-1 AoA Study}

For the evaluation of the NTF203-1 package, a routine operational test of the sensor was conducted with comparisons to the NTF204-1. For this study, both the NTF204-1 and NTF203-1 were tested at three temperatures, $72^{\circ} \mathrm{F},-150^{\circ} \mathrm{F}$, and $-250^{\circ} \mathrm{F}$, in a thermal chamber that is discussed in Section IV. The NTF204-1 is assumed to be the standard of NTF AoA packages, since it is viewed as the best performing package.

Results of the operational testing is presented in Section V, where comparisons between the NTF203-1 and NTF204-1 are made. Shifts of the calibration coefficients due to temperature are evaluated to asses the performance differences between the two packages.

\section{Laboratory Characterization Process (Operational Test)}

Various tests are conducted on AoA packages prior to wind-tunnel operations to ensure proper AoA functionality and data quality. One series of tests ensures the measurement stability of an AoA package for temperatures expected during wind-tunnel operations. For this test a pitch-only calibration is performed at various environment temperatures, typically $72^{\circ}$ and $-250^{\circ}$, and occasionally and intermediate temperature $\left(-150^{\circ}\right)$. The test apparatus for stability testing is shown in Figure 3, where a precision index table is inserted into a temperature controlled chamber. Angle measurement of the table is provided by a Heidenhain ROD800 rotary encoder that has an accuracy of 1 arc-second. Environmental temperature is measured near the AoA package by a thermocouple, which is used to monitor and control the environmental chamber settings.

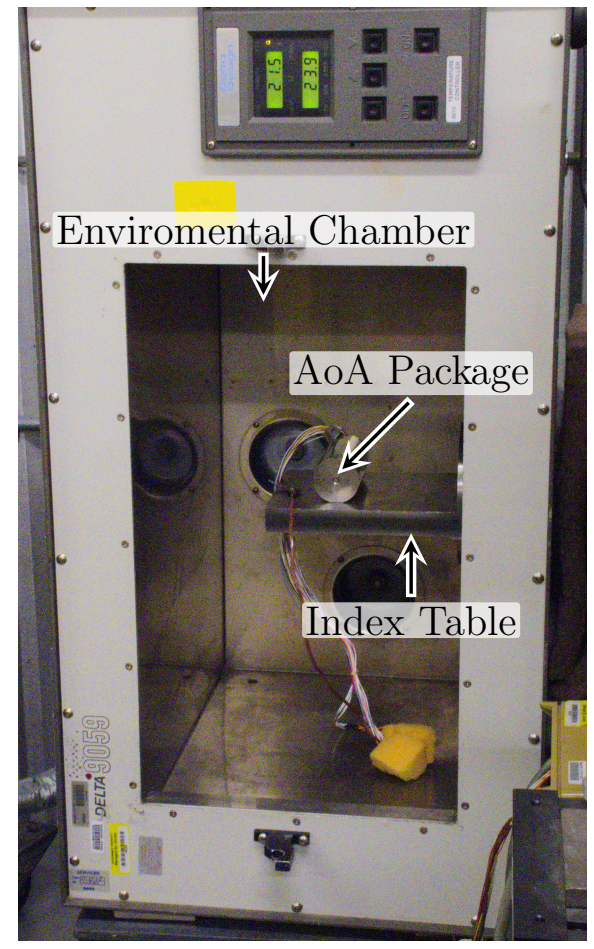

Figure 3: AoA package operational testing apparatus.

Each calibration of the AoA package is conducted over a range of $\pm 0.866 \mathrm{~g}$ in steps of $0.087 \mathrm{~g}$, for each temperature. Additionally, each AoA package is tested in the forward and reverse orientation to remove any warping of the index table, which will be discussed later. Calibration data of each temperature set is used to solve for the calibration coefficients in Equation 1:

$$
\alpha=\sin ^{-1}\left(\frac{V_{q}-b}{S}\right)-\phi
$$


where $\alpha$ is the angle of attack, $V_{q}$ is the Q-Flex voltage, $b$ is the bias, $S$ is the sensitivity, and $\phi$ is the offset angle.

Sensitivity and bias shifts are found by the differences between coefficients for the room temperature and cryogenic conditions. However, the offset shift must be handled differently due to warping of the index table. Forward and reverse offsets are used to estimate the table offset and accelerometer offset by solving:

$$
\begin{aligned}
& \phi_{b}+\phi_{a}=\phi_{\text {Normal }, T=-250}-\phi_{\text {Normal }, T=72} \\
& \phi_{b}-\phi_{a}=\phi_{\text {Reverse }, T=-250}-\phi_{\text {Reverse }, T=72}
\end{aligned}
$$

for the index table shift $\phi_{b}$ and the accelerometer shift $\phi_{a}$.

Ideally, all coefficient should not shift due to environmental temperatures since the packages are internally heated. Slight changes in the coefficients do occur while thermal cycling, and are acceptable when results are within the values listed in Table 1 . These specifications are developed based on the overall angle error that is allowed during cryogenic testing at NTF.

Table 1: Package performance specifications.

\begin{tabular}{cc}
\hline \hline Coefficient & Shift Limits \\
\hline Sensitivity & $\pm 0.04 \%$ \\
Bias & $\pm 20 \mu \mathrm{V}$ \\
Acc. Offset & $\pm 0.005^{\circ}$ \\
\hline \hline
\end{tabular}

Aforementioned, the testing described in this section are used for testing the operational readiness of NTF AoA packages. Calibration coefficient estimates are not transfered to the tunnel data system, instead an in-situ calibration is performed at room temperature when installed into the model. An in-situ calibration is conducted in this manner to remove any offsets that will exist due to the mounting of the AoA package and any model offsets. Since no cryogenic calibration is performed on the sensors in-situ, it is assumed that the cryogenic shifts have no affect on the sensor accuracies.

\section{AoA Cryogenic Shift Results}

The following results will focus on the sensitivity and bias calibration coefficients from the operation tests conducted on both packages. The offset coefficient in Equation 1 will not be studied due to the influence from installation on the index table. In an ideal case, all calibration coefficients will not shift between room temperature and the cryogenic condition, since each package is internally heated to $160^{\circ} \mathrm{F}$ during each of the tests conducted.

Calibration results for both packages, and both internal sensors are listed in Table 2. Note that in this table two room temperatures are listed for each package/sensor combination, since a post cryogenic cycle room temperature run is taken. This extra room temperature run provides indication of thermal hysteresis of the package/sensor. Due to the small changes in the sensitivity and bias coefficients it is difficult to understand the shifts occurring due to temperature.

Plotting of the percent change from room temperature helps illustrate the affect temperature has on the coefficients. Sensitivity shifts for the primary and secondary Q-Flex sensors in both NTF204-1 and NTF203-1 are shown in Figure 4. For the NTF204-1, the primary sensor does not show any large change in sensitivity, whereas the secondary Q-Flex shows a $0.02 \%$ shift at $-250^{\circ} \mathrm{F}$. Sensitivity shift trends appear to be mostly linear, and suggest that during cryogenic conditions the primary sensor should be used over that secondary.

For the NTF203-1, the behavior of between the primary and secondary are reversed from the NTF204-1, where the primary exhibits shift instead of the secondary. This suggests that during operations the secondary sensor should provide more reliable measurements. Also note that the NTF203-1 primary sensor shows more shift that the NTF204-1 secondary, at a maximum shift of $0.028 \%$. These shifts are are still below the required $0.04 \%$ as listed in Table 1 .

Figure 5 shows the bias shift results for the package/sensor combinations in volts. Note that a help line is used to indicate values that are out of the $\pm 20 \mu \mathrm{V}$ tolerance listed in Table 1. It is shown that the NTF204-1 
Table 2: Calibration Coefficients for NTF204-1 and NTF203-1

\begin{tabular}{lrrr}
\hline \hline Sensor & Temp & Sensitivity & Bias \\
\hline 204-1 Primary & 75 & 0.270054 & $5.04 \mathrm{E}-04$ \\
204-1 Primary & -150 & 0.270056 & $5.04 \mathrm{E}-04$ \\
204-1 Primary & -250 & 0.270058 & $5.04 \mathrm{E}-04$ \\
204-1 Primary & 75 & 0.270054 & $5.06 \mathrm{E}-04$ \\
204-1 Secondary & 75 & -0.272323 & $-1.70 \mathrm{E}-04$ \\
204-1 Secondary & -150 & -0.272355 & $-1.66 \mathrm{E}-04$ \\
204-1 Secondary & -250 & -0.272372 & $-1.63 \mathrm{E}-04$ \\
204-1 Secondary & 75 & -0.272322 & $-1.71 \mathrm{E}-04$ \\
203-1 Primary & 75 & 0.282153 & $-8.75 \mathrm{E}-05$ \\
203-1 Primary & -150 & 0.282095 & $-8.69 \mathrm{E}-05$ \\
203-1 Primary & -250 & 0.282075 & $-8.40 \mathrm{E}-05$ \\
203-1 Primary & 75 & 0.282159 & $-8.26 \mathrm{E}-05$ \\
203-1 Secondary & 75 & -0.2677 & $-7.18 \mathrm{E}-04$ \\
203-1 Secondary & -150 & -0.267705 & $-7.14 \mathrm{E}-04$ \\
203-1 Secondary & -250 & -0.267711 & $-7.19 \mathrm{E}-04$ \\
203-1 Secondary & 75 & -0.267699 & $-7.38 \mathrm{E}-04$ \\
\hline \hline
\end{tabular}

does have smaller shift of the bias voltages, approximately $1.7 \mu \mathrm{V}$ and $7.1 \mu \mathrm{V}$ maximums for the primary and secondary sensors, respectively. The NTF203-1 does show higher bias shifts for the secondary sensor with a maximum drift of $-19.5 \mu \mathrm{V}$. Note that this high value is still within the tolerances set for the packages.

To understand the impact coefficient shifts have on the measurement accuracies of the NTF AoA packages, the cryogenic calibrations are applied to the room temperature data. The residual errors between the set angle and estimated angle when using the cryogenic coefficients on room temperature data for the NTF204-1 are found in Figure 6. Note that in this plot, the zero angle offset is removed since the package would be zeroed during tunnel operations. Results for the primary sensor shows minimal residual error, and errors remain well below the $0.01^{\circ}$ accuracy requirement (illustrated by the additional horizontal lines). The secondary sensor on the other had exhibits higher errors as expected by the observed sensitivity shifts. Despite having errors larger than $0.01^{\circ}$, in the typical operational range of $\pm 20^{\circ}$ the sensor performs well within the desired limits.

Applying the same method to the NTF203-1 shows similar results to the NTF204-1, as illustrated by Figure 7. Again the sensor that shifts in the NTF203-1 is opposite of the NTF204-1, with slightly larger errors. These results are as expected due to the observed sensitivity shifts seen in Figure 4. Similar to the NTF204-1, the secondary sensor does not have errors larger than $\pm 0.01^{\circ}$, but the primary sensor does. Again, within the operational range of $\pm 20^{\circ}$ the primary sensor is within the desired accuracies.

\section{Sensor Improvements}

Due to the observed trends that one sensor per package exhibits shifts of the calibration coefficients during cryogenic conditions, improvements to the NTF203-1 were made. The Q-Flex sensors used within these packages have onboard temperature measurement that was not previously connected. It is expected that the sensitivity shifts of the sensors are due to one sensor changing temperature within the package. The NTF2031 was disassembled and the temperature leads were wired to provide the internal sensor temperatures.

During the reconstruction of the NTF203-1, it was found that a insulation foam disk shown in Figure 1 was not installed during prior work. The foam block missing in Figure 8 would originally shield the primary sensor from cryogenic conditions. During the reassembly a new insulation foam disk was fabricated and installed as indicated in the original manufacturing drawings.

\section{Conclusion and Future Work}

In this study it was found that the NTF AoA packages do exhibit small drifts in the calibration coefficients when subjected to cryogenic conditions. The observed sensitivity shifts were not found to originate from 


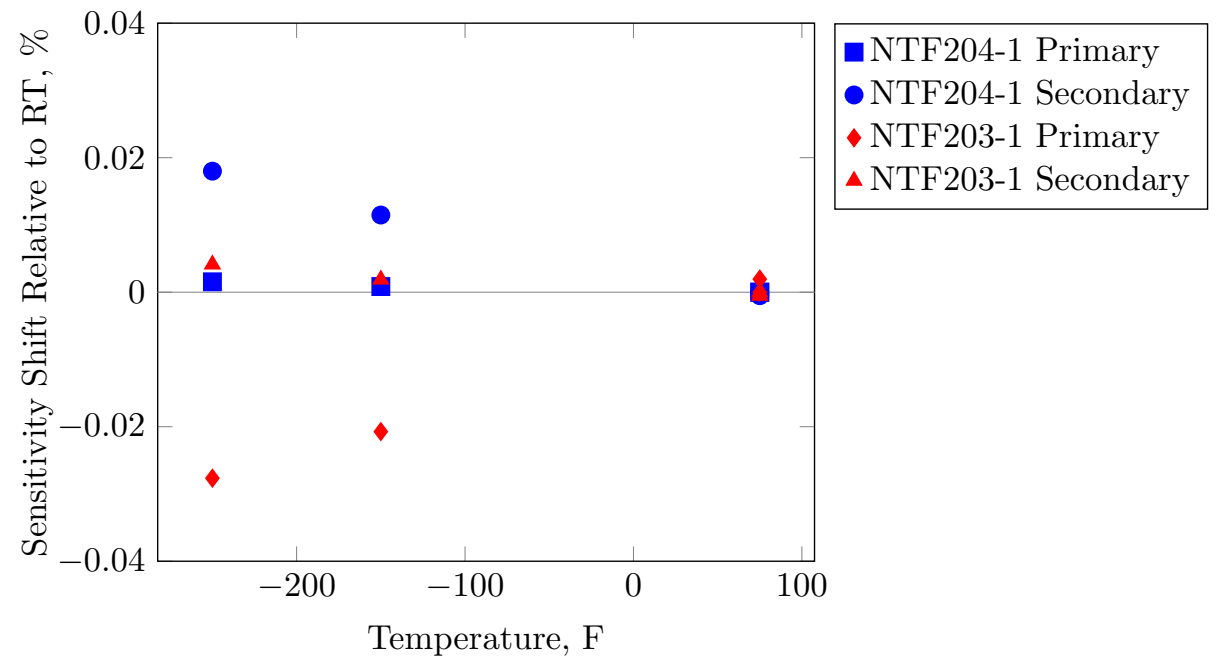

Figure 4: NTF204-1 sensitivity shifts.

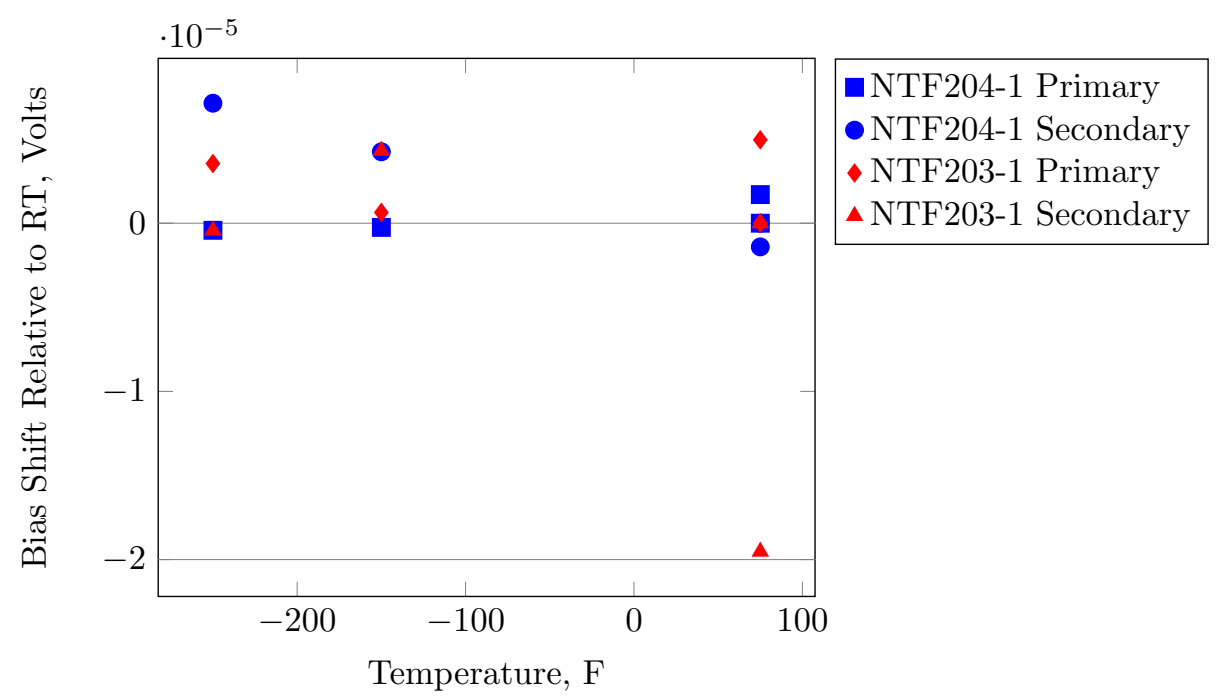

Figure 5: NTF AoA Bias shifts.

the same sensor in both units, secondary for NTF204-1 and primary for NTF203-1. The cross application of the cryogenic calibrations to the room temperature data demonstrated that calibration shifts found do not strongly impact the accuracies of the AoA packages within operational angles. The NTF203-1 primary sensor does show large errors for large angles, but it is strongly suggested that accelerometers should not be used above, or below, $45^{\circ}$.

To better understand the shifts observed in the packages, the individual Q-Flex temperature sensors in the NTF203-1 were connected to be read during further testing. During this process a missing foam insulator was replaced in the NTF203-1. It is expected that the foam replacement will help prevent shifts in the calibration coefficients for the NTF203-1.

Further testing of the NTF203-1 will be conducted to verify if the additional foam core provides better thermal shielding to the primary sensors. The addition of the internal temperature sensors will allow for better diagnostics of the observed shifts. With the inclusion of these temperature sensors, thermal corrections can be made to the accelerometer output, which is recommended by the manufacture for high accuracy use cases. 


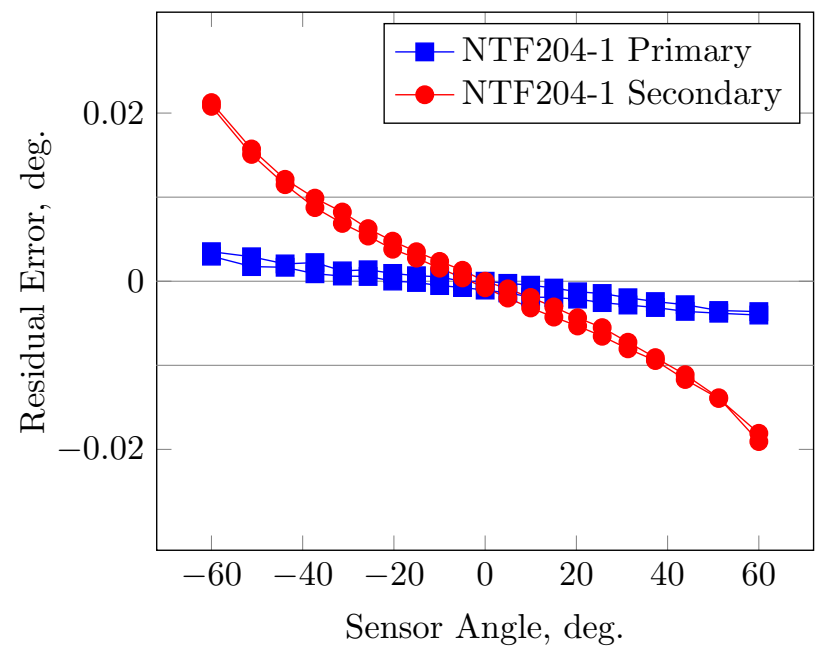

Figure 6: NTF204-1 sensitivity shifts.

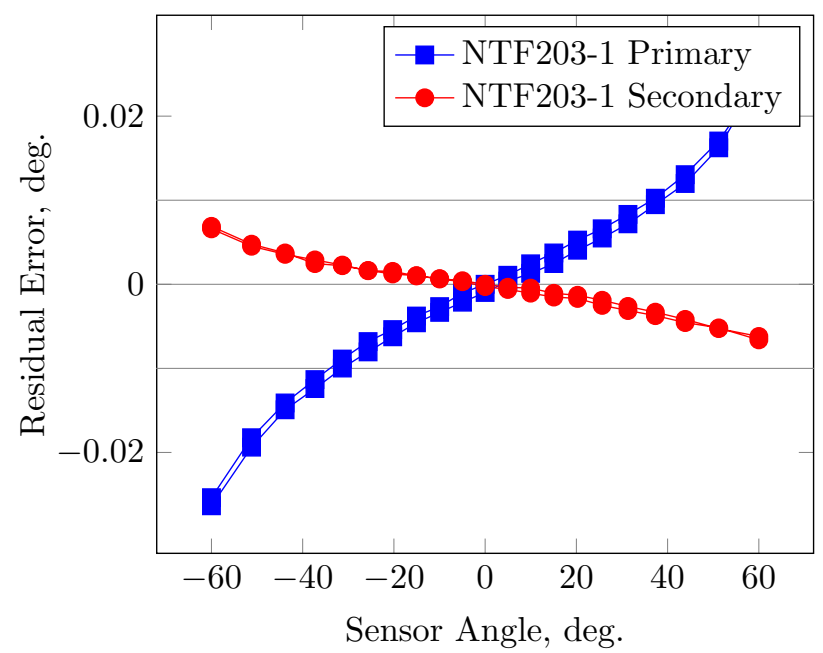

Figure 7: NTF203-1 sensitivity shifts.

\section{References}

\footnotetext{
${ }^{1}$ Holmes, H. K., "The National Transonic Facility," IEEE Aerospace and Electronic Systems Magazine, Vol. 1, No. 2, Feb 1986, pp. 1-7.

${ }^{2}$ Ferris, A. T., "Cryogenic Strain Gage Techniques Used in Force Balance Design for the National Transonic Facility," No. TM 87712, NASA, May 1986.

${ }^{3}$ Crawford, B. L., "Angle Measurement System (AMS) for Establishing Model Pitch and Roll Zero, and Performing Single Axis Angle Comparisons," Aerospace Sciences Meetings, American Institute of Aeronautics and Astronautics, Jan. 2007.

${ }^{4}$ Finley, T. D. and Tcheng, P., "Model attitude measurements at NASA Langley Research Center," Aerospace Sciences Meetings, American Institute of Aeronautics and Astronautics, Jan. 1992.

${ }^{5}$ Quest, J. and Schimanski, D., "Tools \& Techniques for High Reynolds Number Testing - Status \& Recent Improvements at ETW," Aerospace Sciences Meetings, American Institute of Aeronautics and Astronautics, Jan. 2003.

${ }^{6}$ Gorbachev, N. A., Gorbushin, A. R., Krapivina, E. A., and Sudakova, I. A., "Use of accelerometers for measurement of pitch and bank angles in an aerodynamic experiment," Measurement Techniques, Vol. 55, No. 8, Nov 2012, pp. 883-889.

${ }^{7}$ Steinle, F. and Stanewsky, E., "Wind Tunnel Flow Quality and Data Accuracy Requirements," Tech. Rep. AGARD Advisory Report No. 184, AGARD, Nov. 1982.

${ }^{8}$ McDevitt, T. K. and Owen, F. K., "An optical angle of attack sensor," IEEE Aerospace and Electronic Systems Magazine, Vol. 5, No. 2, Feb. 1990, pp. 19-27.
} 


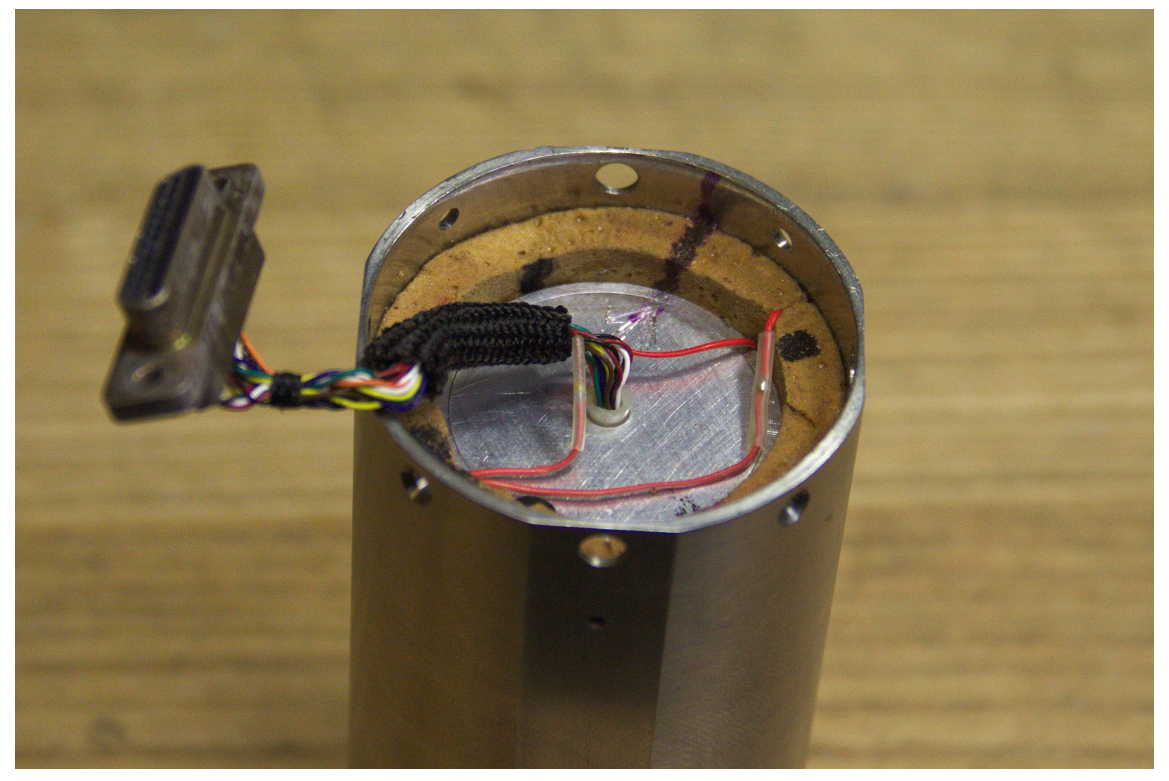

Figure 8: Close-up of NTF203-1 During Disassembly 